\title{
BEAM PROFILE MEASUREMENT WITH FLYING WIRES AT THE FERMILAB RECYCLER RING*
}

\author{
R. Carcagno, Yu. Pischalnikov, J. Krider, M. Hü, E. Lorman, A. Marchionni, S. Pordes, P. Wilson, \\ J. Zagel, Fermilab, Batavia, IL 60510, U.S.A.
}

\begin{abstract}
Flying wires were installed at the Fermilab Recycler Ring for transverse beam profile measurement for both proton and antiproton beams. The following note describes the system configuration, calibration and resolution of the flying wire system, interactions between the wires and the beam, as well as analysis of the transverse beam profile in the presence of a stochastic cooling system.
\end{abstract}

\section{INTRODUCTION}

The Fermilab Recycler is a $3.3-\mathrm{km} 8.9-\mathrm{GeV} / \mathrm{c}$ fixed momentum storage ring located in the Fermilab Main Injector tunnel [1]. Table 1 present relevant Recycler parameters. The Run II Luminosity Upgrade Plan requires the Recycler to play a key role as the repository of large stacks of antiprotons $\left(6 \times 10^{12}\right)$ with the appropriate phase space characteristics to be used in collider stores. For beam stability and efficiency in generating a bright antiproton beam, it is critical to have precise knowledge of the transverse emittances and beam profile. In addition to the existing $1.75 \mathrm{HGz}$ transverse Schottky detector [2], the flying wires were installed during the August 2004 shutdown after thorough vacuum and mechanical tests were performed on the system. The system has been in operation since the start-up in November, 2004.

Table 1: Recycler ring parameters

\begin{tabular}{|l|c|c|}
\hline Parameter & Value & Units \\
\hline Average $\beta$-function, $\beta_{\text {ave }}$ & 30 & $\mathrm{~m}$ \\
\hline Max. dispersion & 2 & $\mathrm{~m}$ \\
\hline Transition, $\gamma_{\mathrm{t}}$ & 20.7 & \\
\hline $\begin{array}{l}\text { Typ. transverse beam } \\
\text { emittances }(\mathrm{n}, 95 \%), \varepsilon_{\mathrm{n}}\end{array}$ & $3-7$ & $\pi \mathrm{mm}-\mathrm{mrad}$ \\
\hline Number of antiprotons & $\leq 6$ & $10^{12}$ \\
\hline
\end{tabular}

\section{SYSTEM DESCRIPTION}

The Recycler flying wire system, which is based on the Flying wire systems installed earlier in the Antiproton Source and the Tevatron [3], consists of two cans (for horizontal and vertical measurements) installed at low dispersion locations. Each can is equipped with an ion pump and a TSP for vacuum maintenance. The cans have been loaded with ferrite for mode damping [4]. The system utilizes rotary motion to move the wires through

\footnotetext{
*Work supported by the Universities Research Assos., Inc., under contract DE-AC02-76CH03000 with the U.S. Dept. of Energy. "martinhu@fnal.gov
}

the beam; the wires are 33 micron monofilament carbon fiber attached to aluminum forks with $133 \mathrm{~mm}$ radius, which are directly driven; the axis of rotation is about 65 $\mathrm{mm}$ from the center of the beampipe. Each measurement consists of moving the wire through 540 degrees of which 180 degrees is constant speed while making two passes through the beam. The DC servo motor is operated in closed loop using a magnetic resolver mounted on its shaft. The resolver signal is converted to 14 bit resolution, which translates to a position resolution of 51 microns. Motion is transferred into the vacuum chamber via a bellows feedthrough to maintain the required vacuum of 0.1 nano-torr. The system is equipped with four scintillation counters ( 2 per plane) to measure both proton and antiproton beams, light produced in the scintillator from secondary particle cascades is read by Hamamatsu R5380 low gain, high current PMT's. The light intensity vs. position data is analyzed and stored on a PC, interfaced with the user through the Fermilab ACNET system. User selectable filters have been installed to attenuate the light intensity by 90 and 60 percent to achieve the required measurement range for the Recycler beam, which is $1 \times 10^{10}$ to $200 \times 10^{10}$ particles. A pulsed LED calibration system is included to monitor the scintillator condition and PMT gain. A 21 slot VME crate handles all the real time data acquisition. It holds the Fermilab specific timing module which can initiate a fly on any decoded clock event, and synchronizes the data acquisition to machine beam synchronous clock. In addition it contains the analog to digital converters (ADC), integrators for the photo multiplier tube signals, and the resolver interface position (RIPFifo) module for each wire. The ADC and RIPFifo modules contain buffers to collect the real time data which are later read into the PC for analysis via the VME to PCI interface slot 0 crate controller. All the instrument settings, readings, and analysis are accomplished on a commercial rack mount PC.

\section{DATA ACQUISITION AND ANALYSIS}

The user loads a specification file for data acquisition, including the timing reference with respect to an accelerator event, a delay from the beam revolution marker, photo-multiplier high voltages and optical filter type. Data acquisition can be triggered with an accelerator event or on demand. A peak/background finding algorithm is applied to fit the intensity vs. position data to a Gaussian plus a linear background. The background data is acquired with each set of data, by fitting a line to points on both sides of the found peak. Figure 1 shows a typical display of a Recycler Flying 
wire measurement, in this case an antiproton beam. The horizontal axis is in millimeter, while the vertical axis is scaled intensity. The analysis program finds the peak and background, performs the fit to acquire the $\sigma$ (the rms beam size for a Gaussian beam) and $\mu$ (beam centroid position), and computes the $95 \%$ normalized emittance based on the input of the local beta function and beam energy:

$$
\varepsilon_{\text {trans. }}(95 \%, \text { normalized })=\frac{6 \pi \sigma^{2}(\beta \gamma)}{\beta_{\text {lattice }}}
$$

Where $\beta_{\text {lattice }}$ is the local beta function, which is based on the design lattice value. The quality of the fit is represented by a chi-square parameter and also observable by the user. Data sets are stored on the PC in a circular buffer available for further analysis.

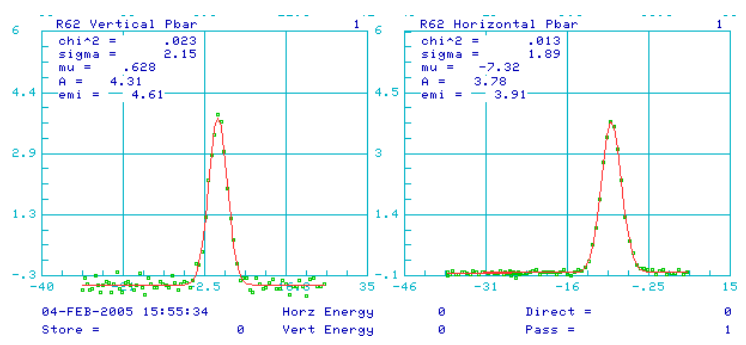

Figure 1: The ACNET program display for the Flying wire measurements of an antiproton beam.

\section{SYSTEMATIC UNCERTAINTIES}

(Peter, please discuss the position uncertainties here, then remove what follows that is unnecessary.)

The position uncertainties due to the resolver and the feedthrough have been estimated in [5] and [6]. The combined uncertainty in position is about $4 \%$ with the current magnetic resolver and feedthrough, or about $8 \%$ in emittance. Uncertainty of the lattice functions at the Flying wire locations also contributes to the calculated emittance (1). BPM Turn-by-turn lattice function measurements [7] have been made to verify that the lattice functions at the Flying wire locations are within a few percent of the design values.

\section{COMPARISON WITH OTHER EMITTANCE MEASUREMENTS}

Initial check on the horizontal scale of the Flying wire measurements were made using local three bumps. The estimated uncertainty in the calculated bump displacement is about $5 \%$. It was observed that the peak displacement measured by the Flying Wires is linear to the three bump amplitude, with a slope of a few percent from unity in both planes (Fig. 2).

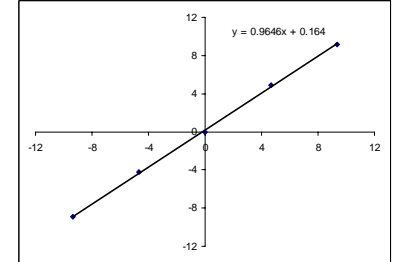

Figure 2: Peak position measured with the horizontal Flying wire vs. calculated three-bump displacement. The slope from the linear fit is 0.965 .

The Flying wire emittance measurements were compared to the $1.75 \mathrm{GHz}$ Schottky detector system previously installed in the Recycler. This detector has been calibrated with a mechanical scraper system and beam. Essentially beam particles were slowly removed with the scraper while data of beam current and scraper position were recorded. From this data the beam profile was reconstructed, and the rms size of the beam can be determined [8]. The tradition at Fermilab is to report the 95\% normalized emittances. The Schottky detector measures the rms size of the beam, and converts it to the $95 \%$ normalized emittance based on the assumption that the beam distribution is Gaussian. The Flying wires measure the profile of the beam and obtains the $\sigma$ from the Gaussian fit. It was verified that the two detectors agreed to within $10 \%$ when measuring a cooled antiproton beam, which has a known Gaussian profile. Furthermore, it was demonstrated that the two detectors diverge when the transverse profile was known to be nonGaussian, with the Schottky detector reporting larger emittances when the distribution had a tail bias (large rms), and the Flying wires reporting larger emittances when the tails of the distribution was truncated (small rms).

During operations the Recycler uses the $1.75 \mathrm{GHz}$ Schottky system for emittance monitoring for all dimensions. The phenomenon if IBS (intrabeam scattering) is an important consideration for the Recycler operation [9]. One of the key utilizations for the Flying wires is the measurement of the transverse emittances when the rms momentum spread of the beam is more than $4 \mathrm{MeV} / \mathrm{c}$, at which point the betatron bands $(\mathrm{n} \sim 20,000)$ overlap and it becomes impossible to measure the band power accurately due to the loss of baseline information.

\section{BEAM-WIRE INTERACTIONS}

Next, we consider the effects on the beam and the wire of passing a relativistic antiproton beam through the carbon wire.

\section{Transverse emittance growth due to multiple Coulomb scattering}

The emittance growth caused by a wire passing through the beam is [10] 


$$
\frac{1}{\pi} \Delta \varepsilon_{N}=3 \gamma \beta \cdot\left(\frac{f \cdot d}{v_{x}}\right) \cdot\left(\frac{13.6 \mathrm{MeV}}{p v_{s}}\right)^{2} \cdot\left(\frac{\pi \cdot d / 4}{L_{r a d}}\right)
$$

Here $v_{\mathrm{s}}$ is the speed of the particles; $d, v_{\mathrm{x}}$ are the diameter and transverse projection of the wire velocity on the beam; $f, p$ are the revolution frequency and momentum of the particles; $L_{\text {rad }}$ is the radiation length of the scattering medium. The calculated emittance growth based on this formula is about $1 \pi$-mm-mrad per operation, or 4 wire crossings of the beam. Actual measurements with the Schottky detector showed about half of the calculated emittance growth, while the Flying wire measurements showed about $30 \%$ of the calculated growth. More accurate knowledge of the composition of the carbon wire is needed to further understand these results.

\section{Ionization energy loss}

The energy loss due to ionization can be directly computed from the known charge number and density of the wire material if the specifics were known. However, a method has been developed in the Recycler to precisely measure the ionization energy loss by fitting to the Landau distribution [11]. Figure 3 shows the logarithmic longitudinal energy spectra of the beam measured with the $1.75 \mathrm{GHz}$ Schottky detector before and after 5 Flying wire operations, a total of 20 wire crossings of the beam. The asymmetry in the final distribution represents an ionization loss of $0.088 \mathrm{MeV}$, which agrees well with a scattering medium with a charge number of 6 and density of $1.78 \mathrm{~g} / \mathrm{cm}^{3}$.

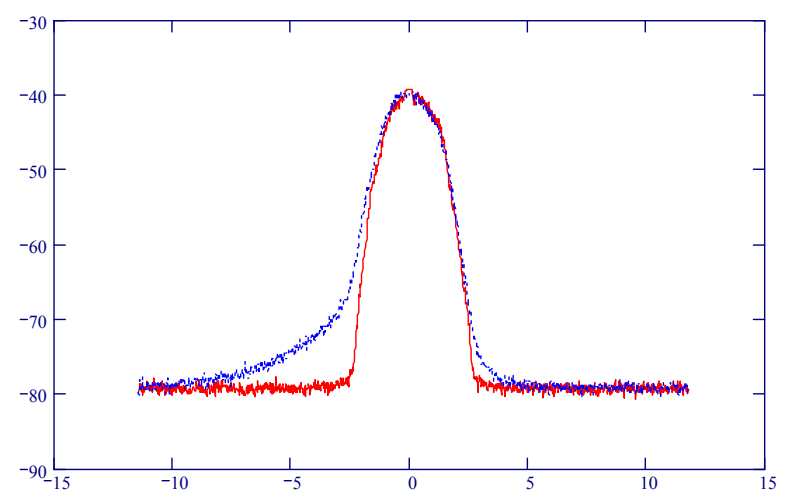

Figure 3: The measured energy spectra of the beam before and after 20 wire crossings of the beam. The vertical axis is on logarithmic scale. The horizontal unit is in $\mathrm{MeV}$. The computed total ionization energy loss based on the analysis of the Landau distribution is $0.088 \mathrm{MeV}$.

\section{Particle loss due to nuclear interaction}

The fractional beam loss during a wire traversal due to nuclear interaction is

$$
\text { Fractional loss }=\frac{\mathrm{f}(\pi \cdot d / 4)^{2}}{v_{x} \cdot L_{\text {int }}}
$$

Here $L_{\text {int }}$ is the nuclear interaction length, and all other variables are defined in (2). For the Recycler Flying wire system this fraction is about $4 \times 10^{-5}$, which for $100 \times 10^{10}$ particles is about 0.56 microamp. This is below the resolution of the Recycler DCCT (Direct Current Transformer), which has a specified resolution of 1 microamp. This amount of beam loss, however, is detectable with the Schottky detector, which can resolve the loss in principle when there is more than $10 \times 10^{10}$ particles in the machine. A study plan has been made to make this measurement when operationally possible.

\section{UPGRADE ITEMS}

An upgrade plan is being considered to improve the performance of the Flying wire system. The main items include smaller wire diameter to reduce emittance growth, better feedthroughs to reduce position uncertainty [5], and more flexible data analysis in which an rms calculation of the beam size is made in addition to the current fitting routine.

\section{CONCLUSION}

The Recycler Flying wire system was installed in August, 2004 and has been in operation since November, 2004. It has been demonstrated that accurate and stable measurements of the beam profile can be made with this system. Due to the measurement induced transverse emittance growth, the frequency of measurements and studies is limited. Future upgrades are being planned to make the system more versatile.

\section{REFERENCES}

[1] Jackson, G., "The Fermilab Recycler Ring Technical Design Report,” Fermilab-TM-1991, Nov. 1996.

[2] Cullerton, E. and Pasquinelli, R., "A $1.75 \mathrm{GHz}$ Schottky Detector for the Recycler," Fermilab RF department technical note, Dec. 2003.

[3] Zagel, J. documents on TeV and pbar FW

[4] Crisp, J., "Beam Impedance of the Flying Wire Can," Fermilab-TM-2212, May 2003.

[5] Wilson, P., "Recycler Flying Wire Status," Fermilab Beams-doc-969-v1, Jan. 2004.

[7] Yang, M. TBT lattice measurement report

[8] Hu, M., "Calibration of the Recycler Transverse Schottky Detectors," Fermilab Beams-doc-372-v2, Jun. 2004.

[9] $\mathrm{Hu}, \mathrm{M}$. and Nagaitsev, S., “Observation of Longitudinal Diffusion and Cooling Due to IntraBeam Scattering at the Fermilab Recycler Ring”, these precedings.

[10] Syphers, M. and Eddy, N., "Emittance Growth Due to Tevatron Flying Wires", Fermilab Beams-doc1199-v2, Sep. 2004.

[11] Hu, M., et al, "Beam-based Vacuum Measurement at the Fermilab Recycler Ring," Fermilab-fn-0758-ad, Aug. 2004. 